\title{
SNEATH, David, Changing Inner Mongolia. Pastoral Mongolian Society and the Chinese State
}

Oxford, Oxford University Press, XIV + 304 p. [Oxford Studies in Social and Cultural Anthropology]

\section{Marie-Dominique Even}

\section{OpenEdition}

\section{Journals}

Édition électronique

URL : https://journals.openedition.org/emscat/1022

DOI : 10.4000/emscat.1022

ISSN : 2101-0013

\section{Éditeur}

Centre d'Etudes Mongoles \& Sibériennes / École Pratique des Hautes Études

\section{Édition imprimée}

Date de publication : 1 mai 2006

Pagination : 427-440

ISBN : 2-9518888-3-X

ISSN : 0766-5075

\section{Référence électronique}

Marie-Dominique Even, " SNEATH, David, Changing Inner Mongolia. Pastoral Mongolian Society and the Chinese State», Études mongoles et sibériennes, centrasiatiques et tibétaines [En ligne], 36-37 | 2006, mis en ligne le 06 avril 2009, consulté le 13 juillet 2021. URL : http://journals.openedition.org/emscat/ 1022 ; DOI : https://doi.org/10.4000/emscat.1022

Ce document a été généré automatiquement le 13 juillet 2021.

(c) Tous droits réservés 


\section{SNEATH, David, Changing Inner Mongolia. Pastoral Mongolian Society and the Chinese State}

Oxford, Oxford University Press, XIV + 304 p. [Oxford Studies in Social and Cultural Anthropology]

Marie-Dominique Even

\section{RÉFÉRENCE}

Changing Inner Mongolia. Pastoral Mongolian Society and the Chinese State, Oxford, Oxford University Press, XIV +304 p. [Oxford Studies in Social and Cultural Anthropology]

1 Inclus dès 1949 dans la république populaire de Chine, les Mongols de Chine ont été, dans les décennies qui ont suivi, tenus à l'écart du reste du monde par le gouvernement communiste, comme les autres populations de ce vaste ensemble issu des conquêtes mandchoues - et comme les Han eux-mêmes. Les diverses politiques menées alors par le régime chinois ont profondément modifié leur environnement politique, social, économique et culturel. Depuis les années 1980, à la suite des réformes entreprises par la nouvelle direction du Parti, le contact avec ces populations et l'accès à l'information les concernant se sont considérablement améliorés. Ils sont encore loin d'être satisfaisants, en particulier pour ce qui est des groupes de population minoritaires (xiaoshu minzu), et restent à la discrétion d'un pouvoir central demeuré très policier et vite enclin à condamner comme séparatiste toute expression identitaire qu'il ne contrôle pas. La nouvelle donne a toutefois permis aux chercheurs de reprendre leurs études sur les peuples non han de Chine et d'éclairer ces quatre décennies d'obscurité. Le livre de David Sneath, qui enseigne l'anthropologie à Cambridge et a co-édité avec Caroline Humphrey plusieurs volumes d'études sur l'évolution du pastoralisme nomade dans ces régions ${ }^{1}$, illustre cette nouvelle génération de travaux. 
Changing Inner Mongolia s'appuie sur des enquêtes de terrain que l'auteur, basé à l'Institut pédagogique de Kökeqota, a menées en 1987-1988 dans les ligues du Silingol et du Hulunbuir, régions choisies pour l'importance de leur population pastorale mongole, puis qu'il a complétées en 1991. Documentation écrite, informations collectées sur le terrain, observations et impressions personnelles constituent le matériau de base de l'ouvrage. Il s'agit pour D. S. de comprendre comment l'État chinois a modelé la vie des éleveurs mongols, ceux-ci étant envisagés non seulement en tant qu'individus mais en tant que sujets politiques, c'est-à-dire comme un groupe minoritaire, de surcroît pastoral, dans une région autonome. À ce titre, l'étude ne s'intéresse pas - l'auteur le précise clairement dès l'abord - aux urbains (des Han pour l'essentiel) et aux Mongols sédentarisés pratiquant l'agriculture.

3 Avant d'entrer dans le vif du sujet, faisons un sort à deux points faibles, qu'il conviendra au lecteur de dépasser pour apprécier l'ouvrage à sa juste valeur. Tout d'abord, les sources. Qui feuillettera les références données en fin de volume constatera qu'à quelques exceptions près elles se limitent à ce qui est disponible en langue anglaise. La documentation existant en chinois et en mongol, ou dans des langues occidentales autres que l'anglais, a donc été peu consultée.Remarquons toutefois que, si réduite soit-elle, cette documentation a été bien utilisée. D. S. a ainsi su tirer profit de publications officielles chinoises sans s'en laisser conter, ou extraire de précieuses données comparatives du travail (en russe) de A. D. Simukov sur la Mongolie du début des années 1930, ainsi que de l'enquête réalisée chez les Mongols de l'Alashan dans les années 1950 - en vue de déterminer s'ils avaient suffisamment rompu avec le féodalisme pour être autorisés à se rattacher à la région autonome de MongolieIntérieure - et parue (en chinois) en 1986.C'est sans pouvoir exploiter au mieux les publications locales qui enrichissent l'approche du domaine mongol, mais en usant avec talent de ses compétences d'anthropologue, que l'auteur a mené son projet. De telles entreprises aboutissent souvent à des résultats mitigés. Ici pourtant, D. S. a traité son sujet avec succès. C'est assez remarquable pour être salué.

4 L'autre point faible, lié à une connaissance du mongol sans doute encore rudimentaire, porte sur les transcriptions des termes mongols. Certes, cette question est un vrai casse-tête et les solutions adoptées ne sont jamais pleinement satisfaisantes. Dans le cadre de ce travail, le choix retenu laisse toutefois le lecteur perplexe. Probablement gêné par l'existence de deux écritures concurrentes - l'écriture oügouro-mongole utilisée en Chine et le cyrillique en usage en Mongolie-, confronté en outre aux particularités phonétiques du mongol de Mongolie-Intérieure, l'auteur emploie des transcriptions très personnelles, peu cohérentes, ignorant souvent l'harmonie vocalique, parfois différentes pour un même exemple, et où se sont glissées maintes coquilles². On rencontre ainsi hüv', aussi erroné qu'inapproprié, pour qubi, courant dans la littérature ; "öröh" au lieu des formes oroh ou oruxu [oruqu], de rigueur si l'auteur avait suivi ses propres "règles " annoncées en début d'ouvrage; “am' öreh", où am' [ami] « vie » est mis pour am [ama] «bouche » (cf. erüke ama « maisonnée, foyer »), qu'il donne comme l'équivalent de $h u$ kou, nom chinois du certificat de résidence, mong. erüke ger-ün dangsa; un curieux "Tayyichi'ut" et un malheureux "Tartar"; des variations sur un même thème, comme torel, törel, töröl; une seule forme bag pour les deux termes, distincts et sans rapport, que sont bag qui désigne l'équipe ou l'unité administrative et baga " petit, jeune »; un "süns ühgüi", essai phonétique qui dénature totalement le nom verbal üheh; ou 
encore (p. 33 note 59) "mörgön tabih" pour mörgöl tabih, le premier ne pouvant en aucun cas signifier, à la différence du second, "accorder une bénédiction». Heureusement, les termes mongols mentionnés sont dans l'ensemble connus, et leur nombre a été volontairement limité. Par ailleurs, ce qui serait moins acceptable dans un ouvrage de mongolisant ne constitue pas, dans ce travail pour le reste solide et destiné à un lectorat plus vaste, un obstacle rédhibitoire.

5 Un peu gênantes également sont les traductions approximatives (telles que Jebstundamba Hutagt, par «Living Buddha », ou celles, pp. 229-230, du chant long évoquant le départ de la fille mariée au loin qu'ont sans doute compliquées les coupures introduites à tort à la fin de chaque vers mongol). On tombera sur quelques explications d'ordre étymologique douteuses (chuulgan, p. 9 note 31 ; höbööd, p. 23 note 16). Dommage, car la démarche en elle-même, bien conduite, peut être éclairante. Ainsi ce höbööd «esclaves" [köbegüd], pris pour le pluriel de höböön «bordure » [köbüge, köbege], alors qu'il s'agit du pluriel de «fils » [köbegün]; cette confusion ôte à D.S. un argument qui aurait pu étayer son analyse par ailleurs pertinente de l'idéologie patriarcale imprégnant les relations sociales des pasteurs nomades. Peut-être faut-il aussi attribuer à une imparfaite maîtrise du mongol l'affirmation (p.37 note 75) que l'expression ah-düü s'applique à l'ensemble comprenant parents proches et amis de même génération qu'Ego ; cela étonne, car elle désigne habituellement les aînés et cadets d'Ego sans qu'ils soient toujours de la même classe d'âge, et d'ailleurs D. S. lui-même, dans le corps du texte, traite de la relation âné-cadet (senior-junior) à propos d'individus appartenant à des générations distinctes.

6 Une relecture par ses collègues et maîtres plus au fait des subtilités de la langue mongole aurait été bienvenue pour éviter ces petits problèmes de forme que la riche information donnée dans ce livre nous fait toutefois bien vite oublier.

7 Les Mongols de Chine, note D. S., ont doublement souffert des politiques de l'État chinois : en raison de leur culture et de leur religion d'une part, mais aussi et surtout du fait de leur mode de production particulier, le pastoralisme nomade. Atypique en Chine, le nomadisme servira justement d'argument à Ulanhu («Fils Rouge »), de son vrai nom Yun Ze, un Mongol toumète sinisé membre de la direction du parti communiste chinois, pour demander des politiques locales adaptées à la spécificité mongole et justifier qu'un rôle central soit laissé aux Mongols dans l'administration de la région autonome. À l'époque où D. S. commence ses observations en MongolieIntérieure, vers la fin des années 1980, le processus de décollectivisation entamé en 1981-1982 - démantèlement des coopératives, retour à une forme de propriété privée, rôle du marché dans les relations économiques - est bien engagé. Cette situation lui permet d'observer dans quelle mesure les efforts naguère entrepris par les autorités communistes pour transformer l'économie pastorale ont porté leurs fruits ou au contraire comment des éléments plus anciens refont désormais surface. On rappellera, s'il en était besoin, que la libéralisation des structures économiques en Chine ne s'est pas accompagnée d'une démocratisation des mœurs politiques. Le fait que D.S. s'intéresse à une population minoritaire et l'objet même de son enquête restent d'ailleurs des sujets suffisamment sensibles aux yeux des autorités pour que l'auteur ait jugé plus prudent d'employer des pseudonymes et de faux noms de districts pour protéger un peu ses informateurs. 
8 Dans ce travail, D.S. s'efforce d'abord de cerner, en les situant dans leur contexte historique, les modalités d'application de la politique socialiste chinoise chez les éleveurs mongols. Puis, à partir du témoignage des protagonistes eux-mêmes et de ses propres observations, il restitue de façon concrète leur vie quotidienne et analyse l'impact sur le système de représentations des choix économiques qui leur ont été imposés et l'arrivée de nouveaux modèles sociaux et culturels entrant en concurrence avec les anciennes valeurs. Le livre est organisé en neuf chapitres - ou plutôt en sept, précédés d'une introduction fournissant le cadre historique général et suivis d'une conclusion récapitulant les processus socio-politiques à l'origine des bouleversements de la période considérée (de 1949 à 1990 environ) et approfondissant quelques-unes des problématiques abordées par l'ouvrage. Les chapitres 2 à 5 (pp. 21-178), consacrés respectivement à la situation sociale et économique dans les steppes de Mongolie-Intérieure avant 1949, aux premières réformes socialistes menées en R.P.C., à la période de la Révolution culturelle et enfin aux années de décollectivisation et de réforme rurale, constituent le volet historique de l'étude. Ce cadre une fois en place, les faits observés par D. S. sur le terrain, le discours qu'il entend et l'analyse qu'il en propose se trouvent prémunis contre les interprétations fantaisistes, les extrapolations et les débordements théoriques qu'une indifférence au contexte engendre parfois chez certains auteurs. Et c'est doté des éléments indispensables à sa compréhension que, pour sa part, le lecteur appréciera le volet plus anthropologique des chapitres 6,7 et 8 qui traitent de l'économie domestique, de la parenté et des clans, et enfin des représentations symboliques.

9 L'auteur porte une attention particulière aux questions d'organisation sociale et économique des nomades mongols. Ainsi, dans le chapitre 2, il souligne la permanence dans la première moitié $\mathrm{du} \mathrm{xx}^{\mathrm{e}}$ siècle de formes de relations socio-économiques similaires à celles de l'époque mandchoue, et ce malgré l'émergence d'une élite intellectuelle et politique et les effets de l'occupation japonaise. Sur la question encore controversée de la nature, féodale ou non de ces relations, D. S. rejoint O. Lattimore et les historiens mongols et considère qu'on a bien affaire, dans cet ordre social rigide et stratifié, à des structures relevant du féodalisme tel que le définit Weber, ou même Bloch. Pour ce qui est du pastoralisme des nomades mongols avant 1949, il en trouve un reflet assez fidèle dans la peinture qu'en fit A. D. Simukov pour la Mongolie des années 1930. Le géographe russe fut en mesure de décrire, a posteriori, le pastoralisme prérévolutionnaire avec ses structures anciennes que le nouvel ordre social en marche n'avait pas encore oblitérées. Il attira l'attention sur la tendance des éleveurs riches à former des campements (les "hoton" ou "hot-ail") avec des familles pauvres pour bénéficier de main-d'œuvre et sur la demande de ces dernières, incapables de survivre seules, pour ce type d'arrangement; au service direct d'un riche, recevant en retour, à la discrétion du propriétaire, nourriture, tissu pour se vêtir, etc., ces familles nomadisaient avec lui : elles avaient le statut de "jarch" [zarcu] «serviteur ", le riche occupant la position d'“ah" [aq-a] ou aîné. Une autre forme de dépendance, indirecte cette fois, n'impliquait pas de nomadiser conjointement; sans dénomination particulière, elle liait un propriétaire de cheptel (riche éleveur ou lama, monastère, institution, etc.) à un éleveur indépendant à qui le premier confiait le soin d'un troupeau en retour d'une partie du produit de l'élevage. D. S. propose de faire appel, pour décrire ces types de relations, à la notion de patron-client, définie comme une relation à long terme, volontaire, fondée sur une inégalité entre les deux parties, relativement informelle mais instaurant de fortes obligations de part et d'autre. Cette notion a l'avantage de rappeler que, si des liens de parenté unissent souvent les 
membres d'un campement, ils sont loin d'être exclusifs. Autres données fort intéressantes sur lesquelles s'appuie l'auteur, celles tirées de l'enquête socio-historique diligentée chez les Mongols de l'Alashan au milieu des années $1950^{3}$ : D. S. observe que les dignitaires laïques et religieux ainsi que leurs proches continuaient, grâce aux réseaux de relations qu'ils entretenaient et à la priorité donnée aux grands troupeaux sur les petits, à se réserver l'usage des meilleures pâtures (surtout celles, cruciales, d'hiver et de printemps) ; l'abolition de leurs privilèges ne paraît pas avoir tellement affecté à l'époque la répartition des terres entre les éleveurs, ni modifié la distribution inégale du cheptel.

Établissant un parallèle avec la position dominante des aînés vis-à-vis des cadets, des hommes vis-à-vis des femmes, l'auteur montre que le concept patriarcal d'autorité présent dans l'organisation familiale imprègne également l'ordre social féodal prérévolutionnaire. Les données Alashan indiquent d'ailleurs que les relations entre un propriétaire mongol et son employé han étaient d'une tout autre nature : commerciales et impersonnelles, avec des conditions d'embauche plus profitables à l'employé, négociées au préalable, limitées dans le temps, ces relations ne s'inscrivaient manifestement pas dans ce réseau de dépendance et de clientélisme paternaliste qui caractérisait alors les rapports entre un " patron » et son « client » mongols. La même logique d'autorité patriarcale, relève D. S., est également à l'œuvre dans les relations que les éleveurs entretiennent symboliquement avec les esprits-maitres des lieux : les faveurs de ces autres "maîtres" se concilient elles aussi à coup d'offrandes et de cadeaux, comme lorsqu'il s'agit d'obtenir le patronage de personnes influentes.

11 Pour D. S., la production de telles inégalités au sein de la société mongole serait donc à imputer au système pastoral mongol lui-même; celui-ci divergerait à cet égard, remarque-t-il, du pastoralisme d'Afrique de l'Est, qui ne permet pas l'émergence de fortes inégalités.

12 Les chapitres 3, 4 et 5 traitent de la façon dont les nomades de la région autonome de Mongolie-Intérieure ont été de plus en plus atteints par les programmes politiques et économiques que la Chine communiste a lancés de 1949 aux années 1990. La mise en place des réformes se fit d'abord de manière relativement souple et graduelle grâce à l'influence d'Ulanhu. Les riches éleveurs continuèrent, durant les premières années de la R.P.C., à employer de la main-d'œuvre, mais sur une base désormais contractuelle. De même, sur le plan religieux, les autorités, pourtant fort méfiantes à l'égard du clergé bouddhique, ne cherchèrent pas à l'éradiquer immédiatement (ce qui n'empêcha pas des attaques contre les monastères, en particulier au cours de la campagne visant les contre-révolutionnaires en 1951). La collectivisation démarra en 1955 et le bétail, hormis quelques têtes laissées à l'éleveur, devint en théorie propriété des coopératives de production, celles-ci se chargeant de répartir les tâches entre les quelque cinquante familles enrôlées et de leur verser un salaire. Même à ce stade qui précéda de trois ans la création des communes populaires, les coopératives préservaient encore, remarque l'auteur, les intérêts des éleveurs les plus riches, payant ces derniers en fonction de leur contribution initiale en cheptel, les autorisant à conserver une bonne part du produit de leurs troupeaux et confiant le soin de ceux-ci aux membres ordinaires de la coopérative : une formule guère éloignée, note D. S., de celle du süreg tabih, placement de troupeaux chez un autre éleveur.

13 Les choses changèrent radicalement avec la mise en place des communes populaires, le Grand Bond en avant et surtout la Révolution culturelle. Le lecteur trouvera beaucoup 
d'informations et de détails concrets sur les formes que prit dans les steppes la désastreuse politique maoïste. Les Mongols de Chine gardent un souvenir très amer de cette période, il est vrai que les victimes furent tout particulièrement nombreuses parmi eux. Le prétexte avancé pour une chasse aux sorcières en règle fut un prétendu parti mongol aux activités secrètes (le parti populaire de Mongolie-Intérieure ou Neirendang, un parti en fait dissous en 1947 selon les informateurs de D. S.); les persécutions affectèrent un adulte mongol sur trois. Les autorités chinoises en profitèrent pour condamner toute expression de l'identité mongole, fût-elle seulement vestimentaire ou rituelle, et pour enlever aux Mongols toute responsabilité véritable dans l'administration de la région autonome. Parallèlement, la période fut aussi marquée par une intensification de la colonisation han en territoire mongol (voir par exemple les tableaux 3.4 et 3.5 comparant l'évolution des populations mongole et han au Hulunbuir entre 1950 et 1970, d'après des statistiques copiées dans les archives locales par un informateur de l'auteur) et par une notable dégradation des pâturages. La lecture de l'étude de D. S. confirme que les Mongols n'ont jamais recouvré depuis, dans leur propre région autonome, le rôle qu'ils avaient tant bien que mal préservé jusqu'au milieu des années 1960. Quant aux conséquences sur leur mode de vie de l'installation de millions de Han en Mongolie-Intérieure et de la politique d'assimilation menée par les autorités chinoises, le mal était fait.

Le chapitre 5, qui clôt le volet historique, est consacré à la décollectivisation (1981-1985) et à la politique de modernisation initiée par Deng Xiaoping. Les éleveurs connurent à nouveau une réorganisation de l'élevage, cette fois sur le principe du transfert de la responsabilité aux familles, qui entraîna rapidement une croissance des revenus (l'auteur souligne à ce propos qu'il convient de ne pas associer augmentation du cheptel et amélioration du niveau de vie: ce dernier dépend du degré de commercialisation et de consommation du bétail décidé par les autorités). Les quotas régissant la production agricole furent partiellement supprimés à partir de 1985, et celle-ci fut dès lors régie par les lois du marché. L'application de ces réformes, loin d'être uniforme, connut de grandes variations d'un endroit à un autre. Les cadres et les officiels tirèrent avantage de leur position pour s'approprier le matériel ou les bâtiments des anciennes communes, les meilleures prairies réservées à la production de fourrage, ou encore les prêts nécessaires à leur installation. La question des pâturages témoigne des tâtonnements de cette période. Dans un premier temps, ils conservèrent leur statut collectif et leur utilisation continua à être régulée par les autorités locales. Mais, dès le milieu des années 1980, une nouvelle politique fut adoptée, visant à réduire le surpâturage (que les autorités attribuaient alors à l'indifférence des éleveurs envers les pâtures communes! D.S. précise que cette explication a été rejetée depuis par une partie des spécialistes): on affecta donc en propre aux éleveurs des pâturages, d'un seul tenant ou bien séparés selon la saison. Or, remarque l'auteur, le déplacement des troupeaux entre les pâtures d'hiver et de printemps et celles d'été et d'automne n'est pas toujours chose aisée, et beaucoup d'éleveurs finissent par laisser leurs troupeaux en permanence dans l'une des zones; dans le cas où il n'y a pas de séparation saisonnière des pâturages, les déplacements sur de longues distances sont devenus inutiles. En conséquence, la mobilité des éleveurs s'est considérablement réduite. D. S. y voit, à l'instar de nombreux éleveurs, l'un des facteurs majeurs de la détérioration continue des pâturages, que, pour leur part, les autorités locales veulent expliquer aujourd'hui par la croissance excessive du cheptel. Celui-ci n'a pourtant pas crû de façon notable par rapport à la fin des années 1920, ce 
sont la superficie des pâturages disponibles et leur qualité qui ont diminué, souligne l'auteur.

15 Le fonctionnement actuel des districts mongols, ou sum, est présenté à travers l'exemple d'un district de la bannière de gauche des Nouveaux-Bargas, dans le Hulunbuir, subdivisé en six gachaa. Le sum constitue le cadre de vie et de relations sociales des éleveurs : ils y travaillent, s'y rencontrent, s'y marient le plus souvent et n'en sortent pas sans bonne raison. D. S. remarque également que les mariages mixtes ne sont pas chose courante parmi les éleveurs et que, même chez les Mongols sédentarisés des régions agricoles, ils restent limités. L'auteur consacre plusieurs pages à la politique de restriction des naissances, que promeut localement la responsable de la Ligue des femmes. Bien que cette politique soit moins stricte à l'égard des populations minoritaires, les Mongols l'acceptent très mal. Les éleveurs parviennent malgré tout à contourner ces restrictions de diverses manières (paiement de l'amende, dérogation, éloignement provisoire).

Les portraits que brosse D.S. de quelques familles du Silingol et du Barga chez lesquelles il a enquêté permettent au lecteur de saisir dans sa globalité la vie de ces éleveurs à la fin des années 1980. L'auteur montre que les différences économiques entre éleveurs sont déjà marquées ${ }^{4}$ et que des formes de relation patron-client sont apparues. Ainsi, l'ancien chef d'une brigade de production habitait une maison en dur (l'ancien centre de brigade) que des ouvriers han étaient occupés à agrandir. Ce communiste fier de ses machines agricoles (qui avaient été celles de la commune populaire) et dédaigneux de la tradition - qu'il s'agisse d'offrir quelques prémices d'alcool aux esprits ou de participer aux cérémonies collectives à l'oboo - possédait plus de 500 têtes de bétail, dont une centaine de bovins et chevaux, qu'il faisait garder par des éleveurs pauvres; il les employait également à faire les foins, en septembre, sur sa riche prairie d'un $\mathrm{km}^{2}$. L'un de ces éleveurs, malgré ses quelque 120 moutons et chèvres, habitait une yourte aux feutres usés; il recevait en échange de ses services un salaire mensuel de 45 yuan, qu'il trouvait insuffisant, et pouvait bénéficier en outre des machines agricoles et du puits de celui «avec lequel il travaillait». C'est par cette expression en effet, et non par le terme darga « chef », souligne D. S., qu'il désignait son patron, de dix ans son aîné. En sens inverse, le terme "jarch" [zarcu] «serviteur », jugé dévalorisant, n'était pas utilisé: on lui préférait, dit l'auteur, celui de "habsragch" [qabsuruүci], que D. S. traduit par «assistant » ou « associé ». Cette attitude témoigne du rejet des hiérarchies et des inégalités très fortes de l'« ancienne société ", martelé par le discours communiste et qui reste, dit l'auteur, encore vif chez les éleveurs actuels. Un autre cas de figure est présenté à travers le portrait d'un éleveur éduqué, persécuté durant la Révolution culturelle, qui avait désormais la garde de 360 têtes, dont 30 bovins et 30 chevaux, appartenant à la branche locale de la compagnie commerciale d'État ; venu d'une autre bannière en 1983, cet éleveur n'avait pas de droits propres sur des pâturages mais utilisait ceux de la compagnie ; il pouvait garder la moitié du croît du bétail et l'intégralité du revenu de la laine, ce qui lui assurait un bon niveau de vie. Là encore, les apparences peuvent être trompeuses, indique D.S.: le fait d'être employé et non propriétaire de ses troupeaux n'a pas forcément la pauvreté pour corollaire.

17 Le chapitre 6, qui nous livre une version concrète et actualisée de la vie quotidienne des éleveurs mongols de Mongolie-Intérieure, ouvre le volet ethnologique. Certes indispensable dans le cadre d'un tel ouvrage, il apportera sans doute moins d'éléments 
nouveaux aux spécialistes du domaine. On notera (p. 187) une tentative de l'auteur de classer le don chez les Mongols selon six contextes (qui incluent la plupart des cas mentionnés par N.L.Zhukovskaya dans sa typologie): hospitalité, marquage de relations de parenté ou d'amitié, marquage de relations économiques, mariage, acte politique, acte religieux. D.S. fait un rapprochement entre les liens que les familles d'éleveurs cultivent auprès des personnages influents, par le biais de cadeaux et d'une généreuse hospitalité, et les réseaux de relations (guanxi) créateurs d'obligations de leurs voisins han. Sur un plan plus strictement économique, l'auteur remarque que ni le système de points de travail individuels introduit par les communes populaires ni la mise en place d'un "marché socialiste" n'ont remis en cause l'ancien modèle de propriété familiale conjointe que gère le chef de famille (celle-ci constituée généralement d'un couple âgé, de ses enfants encore célibataires et d'un enfant marié, occupant le plus souvent deux yourtes voisines) ; au contraire, puisque D. S. ajoute que, les droits de pâture, les machines et les abris à bétail ayant été alloués au départ aux chefs de famille, les fils mariés tendent à demeurer plus longtemps auprès de leur père. La comparaison introduite par l'auteur entre les lignes de dépense des éleveurs mongols en 1925 (à partir des données étudiées par W. Karamisheff) et celles qu'il a pu observer lui-même en 1988 ne témoigne pas de changements profonds; il relève une augmentation du budget consacré à l'alimentation, aujourd'hui plus variée et dont moins de la moitié est assuré par la production familiale (contre les deux tiers en 1925), ainsi que des frais de logement, le feutre des yourtes étant acheté à des artisans chinois plutôt que fabriqué par l'éleveur ; en ce qui concerne la part affectée au culte collectif à l'oboo (une tête de bétail), il note qu'elle n'a pas changé.

18 Le système de parenté et la question des clans fait l'objet du chapitre 7. L'auteur oppose l'existence chez les Bargas de clans (appelés du nom mandchou hala) exogames et patrilinéaires à leur quasi-absence chez les Mongols du Silingol, ces derniers ne connaissant que la noblesse borjigid issue des gengiskhanides, précisément absente chez les premiers. D. S. ne se satisfait pas de l'explication couramment admise selon laquelle la société pré-gengiskhanide connaissait des groupes de parenté territoriaux et que l'apparition d'une noblesse est une conséquence des conquêtes gengiskhanides; il met également en doute la présence avant ces conquêtes d'un système de parenté segmentaire. Ces conceptions lui paraissent en effet influencées par le modèle, établi par Evans-Pritchard à partir des Nuer, de sociétés pastorales où une opposition segmentaire fonctionnant par scission et fusion de lignages remontant à un même ancêtre tend à maintenir une égalité entre eux, et où les alliances politiques sont fondées sur la proximité généalogique. Modèle que les auteurs auraient tendance à projeter sur les nomades mongols, sinon de l'empire mongol, du moins de la période l'ayant précédé. D.S pourtant ne voit pas dans les généalogies ou les alliances mentionnées par l'Histoire secrète de caractère particulièrement segmentaire. Il considère que sous les noms des lignages ou clans cités dans cette chroniquese trouvent probablement davantage de sujets que de membres du lignage, et que ces noms servaient plutôt à désigner des structures politiques dirigées par ces lignages aristocratiques (pp. 200-201). Par ailleurs, on sait que la fondation d'une nouvelle puissance politique par Gengis Khan a permis à son lignage d'occuper une place dirigeante. Il apparaîtrait donc, explique D. S., que seuls les groupes n'ayant pas fait partie de l'État genghiskhanide et chez lesquels la filiation ouvrait encore des droits d'usage des pâturages, comme l'a montré C. Humphrey pour les Bouriates, ont conservé des clans (son premier argument n'est que partiellement vrai : les groupes en question 
- Bouriates, Bargas, Oïrates - ont fait partie de l'empire mongol, puisqu'ils ont été conquis et/ou ralliés par le fils aîné de Gengis Khan, Zöci, mais ils s'en sont ultérieurement détachés tandis que la plupart des Mongols orientaux continuaient à dépendre de l'aristrocratie borjigid). Au vu de la présence de clans chez les Tchakhars, ou même les Toumètes, plus convaincant encore lui paraît être un point relevé par S. Szynkiewicz : dans les premiers temps de leur conquête, les Mandchous ont procédé à l'enregistrement des lignages des populations qu'ils soumettaient, ce qui a constitué une promotion des clans, en particulier dans le cas des populations intégrées dans leur système des Huit Bannières. L'auteur suggère que la place accordée aux clans (rod) chez les Bouriates par l'administration russe a pu pareillement renforcer leur existence. Enfin, sans trop y croire, D. S. suggère que le bouddhisme, avec ses nombreux moines et leur pratique informelle de prendre femme et de procréer en dehors du cadre matrimonial habituel, aurait pu constituer un facteur d'érosion des clans.

Dans la suite du chapitre, l'auteur s'attache à décrire l'organisation des éleveurs telle qu'il l'a observée dans les deux zones étudiées. Les Bargas (plus précisément les Nouveaux-Bargas, bien que l'auteur oublie souvent de le préciser) sont répartis en clans exogamiques ayant pour cadre le district (sum), plus rarement la bannière. Bien que les explications des informateurs au sujet de la fréquence remarquable des mariages entre les deux clans principaux du district ne permettent pas d'y voir une pratique du mariage préférentiel, celui-ci avait déjà été noté par $\mathrm{H}$.Vreeland pour la MongolieIntérieure et parait donc s'être maintenu. Les membres d'un clan se retrouvent chaque année pour le rituel collectif à l'oboo organisé à tour de rôle par un "trésorier » qui collecte auprès de chaque famille sa contribution; D. S. remarque qu'il est la seule manifestation d'autorité formelle au niveau du clan. Le rituel est suivi des festivités habituelles (naadam). La participation à l'un comme aux autres n'est pas réservée aux seuls membres du clan, écrit l'auteur : pourtant, comme il précise aussitôt que les visiteurs ne sont pas considérés comme participants à part entière et qu'un petit clan qui se joindrait au premier pour des raisons pratiques devrait avoir organisé au préalable une " cérémonie spirituelle » distincte à son propre oboo, on en déduira que le rituel à l'oboo et les bénéfices qu'il engendre pour la communauté ont tout de même un caractère exclusif.

Chez les Abaga du Silingol, point de clans ni de longues généalogies, mais une égale importance de la parenté dans la vie quotidienne. La famille étendue y représente l'unité de parenté la plus identifiable. Le mariage est prohibé entre individus apparentés en deçà de la $3^{\mathrm{e}}$ ou $4^{\mathrm{e}}$ génération, et en pratique cette restriction est prise en compte non seulement en lignée paternelle, mais aussi, note l'auteur, en lignée maternelle. Si la résidence est souvent virilocale, l'installation d'un gendre chez son beau-père, pour peu que ce dernier soit riche et manque de bras, n'est pas rare. La consanguinité est un critère important de regroupement, observe l'auteur, mais la composition des campements, loin de refléter un clan en miniature comme l'avait analysé L. Krader, présente une grande flexibilité. Ainsi, l'un des informateurs de l'auteur nomadisait au printemps et à l'automne avec les beaux-parents de sa fille ; son frère, bien que résidant dans la même subdivision administrative, vivait séparément, et il en était de même de deux de ses quatre fils. Les données de A. D. Simukov pour la Mongolie khalkha confirment la fluidité de la plupart des groupes de résidence, mais elles mettent également en évidence la prépondérance des relations de parenté dans les choix de regroupement. D. S. se demande à juste titre si le système d'allocation des pâturages ne tend pas à donner un caractère permanent à ce qui aurait été 
précédemment un regroupement flexible et contingent. On regrettera seulement qu'il n'ait pas fourni d'éléments comparables sur l'organisation des groupes de résidence chez les Bargas.

21 Dans le chapitre 8, intitulé Symbolism and Meaning, plusieurs thèmes retiennent l'attention de l'auteur, dont celui de l'orientation dans la yourte - et plus largement de la logique spatiale - ou encore les manières de se comporter dans un cadre cérémoniel. Tout à fait intéressante est son interprétation des flux d'hospitalité et de service qui parcourent la yourte ou le campement d'est en ouest. D. S. place aussi dans ce chapitre (pp. 226-235) la description d'un mariage barga qui lui sert à illustrer le répertoire symbolique encore utilisé dans les relations sociales. Suit une analyse des cérémonies collectives à l'oboo et des naadam qui les accompagnent (pp.235-249),qui ont officiellement repris en Mongolie-Intérieure en 1981 après avoir été interdites dans les années 1950 et s'être tenues alors, dit-on, clandestinement. Dans ses remarques, l'auteur passe sans doute trop rapidement sur les différences entre ces cérémonies selon qu'elles sont organisées dans le cadre officiel du district ou de la bannière, ou au niveau du groupe de parenté. Ainsi lorsqu'il signale que, dans celles du premier type, ce sont des lamas qui s'approchent les premiers de l'oboo pour procéder aux bénédictions, tandis que, dans le rituel clanique plus fermé - auquel il n'a pu assister, précise-t-il -, c'est un ancien du clan qui invoque en mongol les destinataires des offrandes. Ou encore quand il relève que, si les femmes sont aujourd'hui encore exclues du culte proprement dit, les autorités politiques des districts et des bannières tentent de leur ouvrir davantage les cérémonies organisées à ce niveau en insistant sur l'aspect sportif des festivités. Le seul équivalent au niveau clanique dont l'auteur a eu connaissance concerne un clan nouveau-barga du district voisin, et il résultait de l'initiative d'un ancien cadre. La question de l'exclusion des femmes du cœur du rituel retient l'auteur, qui souligne que ses informateurs n'en donnent aucune explication permettant, par exemple, de l'attribuer à une impureté liée au sang menstruel : ils se contentent de la justifier par la tradition. Par ailleurs, l'interprétation des jeux aux seules fins de célébrer la virilité des hommes que donne I. Kabzinska-Stawarz ne satisfait pas D. S.; pour lui, l'association des hommes à l'activité religieuse et à la politique est ici la plus significative. Quant à une relation avec la nature agnatique des clans exogamiques, elle ne permet pas d'expliquer, selon lui, que l'interdit touche également les cultes à l'oboo qui se tiennent au niveau plus large du district. Cependant, à notre avis, ces rituels seraient les héritiers de ces rituels collectifs que les clans adressaient aux ancêtres, revisités aux siècles passés par un clergé bouddhique intimement associé à l'État centralisé. Du coup, l'exclusion des femmes nous paraît donc bien refléter l'idéologie patrilinéaire du groupe de parenté qui constitue le cadre originel de ces cérémonies placées, rappelons-le, sous l'autorité des anciens du clan, avec ou non le concours des spécialistes religieux. Car, comme l'a d'ailleurs montré l'auteur, le groupe de parenté n'a pas cessé aujourd'hui d'orienter l'organisation des relations dans le cadre restreint du district; son idéologie reste d'autant plus prégnante que le rituel est à l'échelle locale et que la part prise par les institutions politiques et/ou religieuses centralisées est moindre. Quand celles-ci contrôlent la cérémonie, leur idéologie entre en concurrence avec l'idéologie patriarcale qui fonctionne dans le groupe de parenté, quoique les tenants de l'idéologie nouvelle ou dominante restent influencés dans leurs choix et comportements par l'idéologie patriarcale où ils ont grandi.

Dans sa conclusion, l'auteur poursuit sa réflexion sur l'impact que les relations de production socialistes ont pu avoir sur l'idéologie pastorale mongole, et plus largement 
sur la primauté éventuelle du culturel sur le productif. Il avait noté (p. 177) que, si les catégories d'individus occupant les positions structurellement supérieures ou inférieures avaient radicalement changé, la nouvelle réalité économique et politique n'en était pas moins exprimée au moyen de symboles et d'un langage qui lui étaient antérieurs. Son analyse de la situation des éleveurs de Mongolie-Intérieure l'amène à conclure que des structures cognitives, liées de près aux modes d'organisation pastorale, se sont perpétuées dans le domaine domestique en utilisant le langage symbolique traditionnel, alors mêmes qu'elles ne pouvaient plus s'exprimer dans les pratiques politiques et sociales de la période maoïste ; il ne s'agit pas, prend-il le soin de souligner, de simple réactivation ou survivance des modèles culturels anciens, puisque seuls certains éléments se voient «recréés». D.S. cite en exemple les notions de « maître » ou « patron » (ezen), de « règles » ou « usages » (yos), associés à l'organisation familiale, au campement, aux petits regroupements économiques et aux rituels collectifs.

L'auteur reprend, dans cette conclusion, la discussion du système féodal ancien abordée au chapitre 2. Il y voit une version stabilisée des formes de dépendance pastorales et politiques, et il observe que celles-ci sont à nouveau à l'œuvre dans les régions pastorales après la décollectivisation. Pour D. S., si l'émergence d'aristocraties nomades semble bien être le produit de conquêtes guerrières, il ne lie pas forcément le développement de structures étatiques chez les nomades à l'incorporation de sociétés sédentaires en leur sein, comme le fait A. M. Khazanov. Il insiste au contraire sur les processus économiques intrinsèques permettant l'accumulation de cheptel entre les mains d'une élite. Il souligne en particulier le rôle joué à cet égard par la pratique du süreg tabih, qui permettait au propriétaire d'étendre ses possessions sans limitation de main-d'œuvre ou de pâturage et d'accroître ainsi son réseau de dépendants. Cette pratique, qui pourrait être considérée comme corollaire d'une organisation étatique, lui paraît avoir existé, d'après l'interprétation qu'il fait de certains éléments de l'Histoire secrète, avant même la fondation d'un État par Gengis Khan. Plutôt que de parler de relations de classe antagonistes, il préfère interpréter ces rapports en termes de relations de dépendance de type clientéliste et paternaliste. D.S. introduit à cette occasion une comparaison avec les Kirghizes du Pamir afghan étudiés par M. Shahrani ${ }^{5}$, qui lui servent à illustrer le chaînon manquant entre relations pastorales de dépendance clientélistes et féodalisme. Ces Kirghizes sont en effet passés de relations égalitaires à un état de différentiation sociale et économique avéré dans les années 1970 , avec $4 \%$ des familles possédant $85 \%$ du petit bétail ; le khan, garant de l'ordre au sein de la société, y est choisi pour sa richesse, source d'influence et gage d'efficacité. Conscient qu'une évolution "féodale » similaire est impossible dans une MongolieIntérieure désormais trop intégrée dans la Chine, l'auteur se demande toutefois si le développement de telles inégalités matérielles et de ce qu'il nomme des «relations sociales complémentaires" n'est pas un phénomène inhérent à certaines sociétés pastorales. Il serait certainement très intéressant de confronter le schéma proposé par D. S. avec les nouvelles relations socio-économiques qui se sont développées depuis une quinzaine d'années dans les steppes de la république de Mongolie, et de comparer les stratégies employées par les éleveurs de part et d'autre de la frontière (la comparaison a toutefois ses limites en raison de la situation particulière des Mongols de Chine, soumis à une forte présence han sur leurs territoires et qui ne sont plus maitres de leur destin). 
Comme on le voit, l'auteur aborde dans cet ouvrage nombre de sujets passionnants, qu'il nourrit d'observations concrètes et de comparaisons tant diachroniques que synchroniques. La réflexion théorique est riche et pertinente, son expression simple et claire. On appréciera qu'il ait choisi de mener son enquête dans deux communautés d'éleveurs présentant des caractéristiques propres, ce qui lui permet à la fois d'enrichir et de nuancer les données qu'il livre ici. Et l'on se réjouira en particulier de l'éclairage bienvenu que son travail jette sur la Mongolie-Intérieure. Remercions donc l'auteur d'avoir mis à la disposition du lecteur occidental une présentation aussi détaillée et vivante de l'histoire socio-économique des nomades mongols sous le régime communiste chinois, histoire malheureusement encore trop peu connue.

\section{NOTES}

1. C. Humphrey et D. Sneath (éd.), Culture and Environment in Inner Asia (Cambridge, White Horse Press, 1996), 2 vol.; C. Humphrey et D. Sneath (éd.), The End of Nomadism ? Society, State and the Environment in Inner Asia (Durham, NC, Duke University Press, 1999).

2. Maladroites aussi sont certaines transcriptions de termes tibétains, pour lesquels il aurait été préférable de s'en tenir aux formes simplifiées, par exemple d'écrire Sakyapa (d'après la translittération usuelle Sa skya pa ) au lieu de « Sa-gya-pa ».

3. Mengguzu shehui lishi diaocha (Huhehaote, Nei Menggu renmin chubanshe, 1986). L'auteur souligne l'intérêt de ce document, plus objectif que bien des publications chinoises.

4. S'appuyant sur les chiffres d'un district du Silingol, D. S. indique que le pourcentage de ceux possédant jusqu'à 200 têtes est de $40 \%$, et que celui des propriétaires de 200 à 500 têtes est de $50 \%$. Ils ne sont plus que $10 \%$ à en posséder plus de 500 . Il n'a pu obtenir en revanche aucun chiffre ni estimation concernant les familles les plus démunies, qu'il évalue à une sur dix, voire une sur cinq.

5. M. Shahrani, The Kirghiz and Wakhi of Afghanistan: Adaptations to close frontiers (Seattle, University of Washington Press, 1979). 